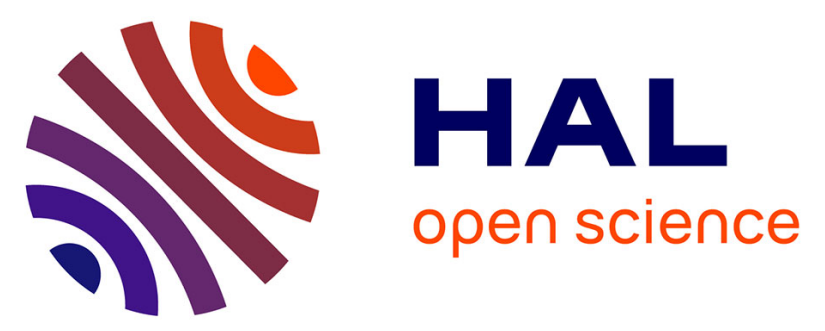

\title{
Syndrome CMMRD (déficience constitutionnelle des gènes MMR) : bases génétiques et aspects cliniques
}

Bruno Buecher, Marine Le Mentec, François Doz, Franck Bourdeaut, Marion Gauthier-Villars, Dominique Stoppa-Lyonnet, Chrystelle Colas

\section{To cite this version:}

Bruno Buecher, Marine Le Mentec, François Doz, Franck Bourdeaut, Marion Gauthier-Villars, et al. Syndrome CMMRD (déficience constitutionnelle des gènes MMR): bases génétiques et aspects cliniques. Bulletin du Cancer, 2019, 106, pp.162 - 172. 10.1016/j.bulcan.2018.10.008 . hal-03486683

\section{HAL Id: hal-03486683 https://hal.science/hal-03486683}

Submitted on 20 Dec 2021

HAL is a multi-disciplinary open access archive for the deposit and dissemination of scientific research documents, whether they are published or not. The documents may come from teaching and research institutions in France or abroad, or from public or private research centers.
L'archive ouverte pluridisciplinaire HAL, est destinée au dépôt et à la diffusion de documents scientifiques de niveau recherche, publiés ou non, émanant des établissements d'enseignement et de recherche français ou étrangers, des laboratoires publics ou privés.

\section{(ㅇ)(1) $\$$}

Distributed under a Creative Commons Attribution - NonCommerciall 4.0 International 


\section{Syndrome CMMRD (déficience constitutionnelle des gènes MMR) : bases génétiques et aspects cliniques}

Constitutional MMR deficiency: genetic bases and clinical implications

\section{Bruno Buecher', Marine Le Mentec ${ }^{1}$, François Doz ${ }^{2,3}$, Franck Bourdeaut $^{2,4}$, Marion Gauthier-Villars ${ }^{1}$, Dominique Stoppa Lyonnet $^{1,3,4}$, Chrystelle Colas ${ }^{1,5} *$}

1. Département de Génétique, Institut Curie, Paris Sciences Lettres Research University, Paris, France

2. Centre d'oncologie SIREDO (soins, innovation, recherche en cancérologie de l'enfant, l'adolescent et l'adulte jeune) Institut Curie, Université Paris Descartes, Paris, France.

3. Université Paris Descartes Sorbonne Paris, France

4. Unité INSERM U830, Institut Curie Paris, France

5. Sorbonne Universités, UPMC Univ Paris 06, INSERM, CNRS, Centre de Recherche Saint-Antoine, Paris, France

* Pour correspondance : Chrystelle Colas

Département de génétique Institut Curie 26 rue d’Ulm 75005 PARIS, France chrystelle.colas@curie.fr 


\title{
Syndrome CMMRD (déficience constitutionnelle des gènes MMR) : bases génétiques et aspects cliniques
}

\author{
Constitutional MMR deficiency: genetic bases and clinical \\ implications
}

\section{Résumé}

La présence d'une mutation constitutionnelle mono-allélique de l'un des 4 principaux gènes MMR est responsable du Syndrome de Lynch qui prédispose au développement à l'âge adulte de tumeurs principalement colorectales et endométriales caractérisées par une instabilité des microsatellites (phénotype $\mathrm{MSI}$ ). Les personnes porteuses de mutations constitutionnelles à l'état bi-allélique de l'un de ces gènes, développent précocement, le plus souvent dès l'enfance, des tumeurs multiples. Cette prédisposition héréditaire récessive est appelée syndrome CMMRD (Constitutionnal Mismatch Repair Deficiency). Le spectre tumoral est différent de celui du syndrome de Lynch. Les tumeurs cérébrales sont aussi fréquentes que les atteintes digestives et des hémopathies sont rapportées dans un peu plus d'un tiers des cas. Ces patients ont également des signes similaires à ceux d'une neurofibromatose de type 1 , en particulier des taches café au lait. Le gène le plus fréquemment impliqué est PMS2 suivi de MSH6, et plus rarement $M L H 1$ et MSH2. Les tumeurs digestives de ces patients sont de type MSI alors que les tumeurs cérébrales peuvent être stables. Du fait d'une présentation clinique variable et de chevauchements phénotypiques avec d'autres syndromes, le syndrome CMMRD est encore mal connu des cliniciens et son incidence est certainement sous-estimée. Une meilleure définition et diffusion des critères cliniques et le développement de méthodes diagnostiques devraient améliorer l'identification de ces patients dès la première atteinte tumorale, voire même avant. Cela permettrait de guider les choix thérapeutiques mais également de mettre en place une surveillance ciblée sur les autres risques tumoraux, pour les patients eux-même mais également leurs apparentés.

Mots-Clefs: mutations MMR bi-alléliques; Constitutional Mismatch Repair Deficiency, tumeurs cérébrales; hémopathies ; cancer colorectal ; neurofibromatose de type 1

\section{Summary}

Inherited mono-allelic mutation in one of the 4 major MMR genes results in Lynch syndrome which predisposes, in adulthood, mainly to colorectal and endometrial tumors characterized by microsatellite instability (MSI phenotype). Individuals with bi-allelic mutations of one of these genes developed early and multiple malignancies, most often in childhood. This recessively inherited condition is named CMMRD for Constitutional Mismatch Repair Deficiency. The spectrum of tumors is distinct from Lynch Syndrome. Malignant brain tumors 
are at least as frequent as gastrointestinal tumors and in more than a third of cases haematological malignancies were also reported. Patients also displayed clinical features similar of neurofibromatosis type 1, especially café au lait spots. The most commonly involved genes are PMS2 and MSH6 while bi-allelic MLH1 and MSH2 mutations are rare. The digestive tumors of these patients show MSI whereas the brain tumors can be "microsatellite stable". Because of variable clinical presentation and phenotypical overlaps with other cancer syndromes, CMMRD syndrome is frequently unrecognized by clinicians and its incidence is almost certainly underestimated. A better knowledge of clinical criteria and diagnosis methods should improve the identification of these patients at least at the time when they develop their first tumor or even before. This will allow adjusting treatment modalities and offering surveillance strategies of other tumor risks, not only for patients themselves but also for their relatives.

Keywords: bi-allelic MMR mutations; Constitutional Mismatch Repair Deficiency; cerebral tumors; lymphoma; colo-rectal cancer; Neurofibromatosis type 1

\section{Introduction}

La présence d'une mutation constitutionnelle à l'état hétérozygote de l'un des 4 principaux gènes du système de réparation des mésappariements de I'ADN ou Mismatch repair (MMR), que sont MLH1, MSH2, MSH6, ou PMS2, est responsable d'un Syndrome de Lynch, qui prédispose au développement à l'âge adulte de tumeurs principalement colorectales et endométriales. En 1999, deux équipes ont rapporté le phénotype clinique de deux patients, homozygotes pour une mutation constitutionnelle du gène $\mathrm{MLH} 1$, issus de mariages consanguins au sein de familles de Syndrome de Lynch. Ces patients avaient des hémopathies dans la petite enfance et l'un d'eux une tumeur cérébrale [1,2]. Ils avaient également une atteinte clinique ressemblant à celle d'une neurofibromatose de type 1 (NF1). Depuis, plus de 200 patients, enfants ou jeunes adultes, porteurs de mutations bialléliques d'un des 4 gènes MMR impliqués dans le Syndrome de Lynch ont été rapportés [35]. Cette prédisposition héréditaire récessive est maintenant considérée comme un syndrome de prédisposition aux tumeurs pédiatriques distinct du Syndrome de Lynch appelé CMMRD pour Constitutionnal Mismatch Repair Deficiency (OMIM \#276300). D'autres appellations peuvent être utilisées telles que bMMRD (biallelic Mismatch Repair Deficiency) ou Mismatch Repair Cancer syndrome. Bien que cela n'ait pas été prouvé sur un plan moléculaire, il est probable, a posteriori, que Jacques Turcot en 1959 ait décrit les premiers cas de CMMRD lorsqu'il a rapporté le cas d'une fratrie avec de multiples adénomes coliques, 
un cancer du côlon et une tumeur cérébrale [6]. Ultérieurement ont été décrits sous le terme "syndrome de Turcot", non seulement des patients probablement atteints de CMMRD, mais aussi des patients atteints de polyposes avec des tumeurs cérébrales et des mutations germinales du gène $A P C$ [7]. Le syndrome CMMRD et le syndrome Turcot ne sont donc pas synonymes mais se chevauchent. L'identification précoce des patients CMMRD, dès la première atteinte tumorale, voire plus tôt, est un enjeu crucial. En effet, elle permet de guider les choix thérapeutiques mais également de mettre en place une surveillance pour les autres risques tumoraux, pour le patient mais également pour ses apparentés.

\section{Caractéristiqu?s cliniqu?}

Les patients atteints de syndrome CMMRD peuvent développer simultanément ou successivement différents types de tumeurs, le plus souvent dès l'enfance (Figure 1). L'âge médian au diagnostic de la première tumeur est de 7,5 ans (extrêmes : 0,4-39 ans) [4]. La durée médiane de survie après le diagnostic de la première tumeur est inférieure à 30 mois et la plupart des patients décèdent avant l'âge adulte [3].

Le spectre tumoral du syndrome CMMRD est différent de celui du Syndrome de Lynch. En effet, les tumeurs cérébrales sont aussi fréquentes que les atteintes digestives et des hémopathies sont rapportées dans un peu plus d'un tiers des cas (Figure 2). L'âge médian au diagnostic des hémopathies et des tumeurs cérébrales est estimé à 6,6 et à 10,3 ans respectivement [3]. Les tumeurs cérébrales correspondent principalement à des gliomes de haut grade mais quelques lésions de bas grade, à très fort risque de transformation en lésions de haut grade ont été rapportées [5]. Les médulloblastomes et les autres tumeurs embryonnaires du système nerveux central sont les autres atteintes tumorales cérébrales les plus fréquentes. Les données moléculaires récentes concernant ces tumeurs cérébrales associées au CMMRD suggèrent qu'elles ont un taux exceptionnellement élevé de mutations somatiques en raison de la conjonction de la déficience constitutionnelle MMR et des mutations somatiques des polymérases inactivant leur capacité de relecture [8].

Les hémopathies les plus fréquemment observées sont des lymphomes non Hodgkiniens (LNH) et particulièrement des LNH lymphoblastiques de type T (LLT). Des leucémies aiguës lymphoblastiques de type T (LAL-T), des leucémies aiguës myéloblastiques (LAM) et des lymphomes de type $B$ sont aussi décrits $[3,4,9]$. La prédisposition aux hémopathies des 
patients CMMRD rappelle le phénotype tumoral des modèles murins MMR-déficients suggérant que ces souris pourraient être de bons modèles pour améliorer les connaissances sur les effets d'une déficience constitutionnelle du système MMR [10,11].

Les cancers colorectaux (CCR) sont les tumeurs du spectre du Syndrome de Lynch les plus fréquentes chez les patients CMMRD mais des tumeurs de l'intestin grêle, de l'estomac, de l'endomètre, des ovaires et des voies urinaires ont également été décrites [3,4]. Toutes ces tumeurs surviennent beaucoup plus précocement que celles observées dans le syndrome de Lynch. Des CCR sont décrits dès l'enfance avec le cas le plus jeune décrit à 8 ans, alors que des polypes adénomateux ont été identifiés dès l'âge de 6 ans. Les cancers du grêle sont également rapportés à un âge très jeune (âge médian : 18 ans ; extrêmes : 7-33) [10]. La plupart des patients CMMRD publiés ont des adénomes multiples du tractus digestif, souvent en dysplasie de haut garde et donc associés à un fort potentiel de dégénérescence. Des adénomes du côlon et du rectum sont signalés chez 67 \% d'entre eux lors de la première coloscopie et ensuite chez $100 \%$ de la cohorte au cours de la surveillance coloscopique [10]. Quasiment tous les patients développent des polypes multiples ou une polypose de type atténuée avant l'âge de 30 ans. Lorsqu'il est inaugural, ce phénotype fait discuter les diagnostics différentiels de polypose liée au gène APC (Polypose Adénomateuse Familiale) ou aux mutations des gènes POLE ou POLD1 (syndrome PPAP, Polymerase Proofreading Associated Polyposis). Ces gènes sont le plus souvent étudiés parallèlement aux gènes MMR ou secondairement lorsqu'aucune mutation des gènes MMR n'a été mise en évidence [11]. Des adénomes de l'intestin grêle et du duodénum sont également rapportés $[3,10]$.

D'autres types tumoraux ont été rapportés chez quelques patients CMMRD. Des neuroblastomes et des tumeurs de Wilms surviennent dans la première décennie. Des sarcomes (ostéosarcome, rhabdomyosarcome, dermatofibrosarcome), des tumeurs génitourinaires sont également décrits [3]. Plusieurs patients ont développé des pilomatricomes multiples (pilomatricomatose), tumeurs bénignes cutanées développées aux dépens des cellules matricielles du follicule pileux [3,12].

Des manifestations non tumorales sont également présentes au premier rang desquelles des éléments pouvant faire évoquer une NF1, en particulier des taches café au lait (TCL). Ces dernières sont le plus souvent atypiques avec des bordures plus irrégulières qu'au cours de la NF1. Elles sont très généralement multiples $(\geq 2)$ mais n'atteignent pas toujours le seuil de 
6 retenu pour le diagnostic de NF1 [13]. Plusieurs patients présentent d'autres signes diagnostiques décrits également dans la NF1 comme des lentigines axillaires, des neurofibromes cutanés simples ou plexiformes, des nodules de Lisch, des pseudarthroses tibiales et, pour un patient, un gliome optique [13]. D’autres éléments cliniques rapportés de façon récurrente sont des tâches hypo-pigmentées, des cavernomes, des agénésies du corps calleux, une immunodéficience modérée avec une baisse des Immunoglobulines (Ig) [4]. En effet, la déficience MMR impact le phénomène de recombinaison des Ig qui se traduit par une diminution ou une absence d'IgG2, IgG4 et d'IgA associée à une augmentation du taux des IgM [14,15]. Ce dernier point est cependant très inconstant car une étude récente de 15 patients atteints de CMMRD n'a pas retrouvé d'éléments cliniques ou biologiques en faveur d'une immunodéficience primaire mais a confirmé la présence d'anomalies des mécanismes de commutation de classe (CRS) et d'hypermutation somatique (SHM) initialement décrits dans ce syndrome [16].

\section{Asp? ?cts génétiqu? $s$}

Le mode de transmission du syndrome CMMRD est autosomiques récessif. Des variants pathogènes bi-alléliques sont rapportés dans les 4 gènes MMR associés au syndrome de Lynch: MSH2, MLH1, MSH6 et PMS2. La répartition des mutations de ces 4 gènes est cependant inverse de celle observée dans le Syndrome de Lynch (Figure 3) : les gènes les plus fréquemment impliqués dans le CMMRD sont PMS2 de façon la plus fréquente, suivi de MSH6 ; les mutations bi-alléliques de MSH2 et de MLH1 sont rares [3-5]. Cette distribution est probablement le reflet de la fréquence des mutations de ces gènes à l'état hétérozygote dans la population générale à la différence de ce qui est observé au cours du syndrome de Lynch, dans la mesure où l'identification des patients est dépendante des pénétrances qui sont plus faibles pour les mutations hétérozygotes des gènes MSH6 et PMS2 [17,18]. Par ailleurs on peut faire l'hypothèse d'une plus grande sévérité allant jusqu'à la létalité des formes bi-alléliques des mutations $M L H 1$ ou $M S H 2$ qui pourrait rendre compte d'une partie Les mutations identifiées chez les patients CMMRD sont des mutations " perte de fonction » ou inactivatrices et la plupart sont de type tronquant car introduisant un codon stop. Elles sont associées à une perte d'expression et de fonction de la protéine correspondante. Cependant environ un tiers des variants décrits sont de signification inconnue (VSI) ; plus 
souvent sur MLH1 et MSH2 que sur MSH6 et PMS2 [19]. II est possible que ces variants permettent le maintien d'une fonction résiduelle de la protéine MMR correspondante $[3,4,19]$. La rareté de ce syndrome et ce taux élevé de mutations potentiellement hypomorphes (c'est-à-dire résultant en une activité protéique réduite mais non nulle) parmi les patients compliquent l'étude des corrélations génotype/phénotype. Néanmoins, on observe que les hémopathies surviennent plus fréquemment chez les patients porteurs de mutations bi-alléliques $M L H 1$ ou $M S H 2$ et, qu'à l'inverse, les tumeurs cérébrales et les tumeurs du spectre du Syndrome de Lynch sont plus prévalentes parmi les patients avec mutations bi-alléliques de PMS2 $[3,19]$. Lorsque l'on s'intéresse à l'âge au diagnostic de la première tumeur, les patients présentant des mutations bi-alléliques de $M L H 1$ ou de $M S H 2$ sont ceux atteints les plus précocement. La proportion de patients atteints d'une seconde tumeur est plus élevée parmi les patients porteurs de mutations bi-alléliques de PMS2 [3] ce qui est probablement à mettre sur le compte d'une plus grande probabilité de survie après la première atteinte et va dans le sens des observations de phénotypes globalement plus sévères et plus précoces pour les patients porteurs de mutations bi-alléliques sur MLH1 ou MSH2

Contrairement au Syndrome de Lynch, les antécédents familiaux sont le plus souvent non contributifs, bien que les deux parents du cas index soient porteurs obligatoires d'une mutation hétérozygote et donc d'un syndrome de Lynch (Figure 1) [3]. Les pénétrances (c'est-à-dire les risques tumoraux) associées aux mutations mono-alléliques des gènes MSH6 et PMS2 sont plus faibles que celles associées aux mutations des gènes MLH1 et MSH2. Ces derniers gènes étant moins souvent impliqués dans le syndrome CMMRD, il n'est pas étonnant d'observer que les parents d'un enfant CMMRD sont souvent indemnes. Dans les maladies récessives telle que le CMMRD, la consanguinité peut augmenter le risque de mutations bi-alléliques. Le taux de consanguinité varie selon l'origine géographique. Si une partie des patients ayant un syndrome CMMRD sont effectivement issus de familles avec consanguinité et porteurs de la mutation causale à l'état homozygote, la majorité des cas rapportés sont hétérozygotes composites et issus d'unions « non consanguines » [3].

Le risque cumulé de cancer, toutes localisations confondues, au cours du syndrome CMMRD semble extrêmement élevé même s'il n'est pas encore évalué de façon fiable et précise. II est exceptionnel d'observer des personnes porteuses de mutations bi-alléliques indemnes 
de cancer au-delà de 30 ans $[3,4]$. Les données actuellement disponibles sont en faveur d'un continuum clinique allant de formes de CMMRD "moins sévères" qui ressemblent à des Syndrome de Lynch avec une atteinte tumorale précoce, à des syndromes de Lynch avec atteinte précoce qui miment des CMMRD $[3,20,21]$. Les bases moléculaires de ce continuum restent à élucider.

\section{Méthod?s diagnostiqu?}

\section{Score clinique}

Le diagnostic de syndrome CMMRD que ce soit chez une enfant ou un jeune adulte a des implications importantes en termes de prise en charge pour le patient lui-même mais également pour l'ensemble de sa famille. Aujourd'hui encore le diagnostic de ce syndrome est souvent retardé voire non fait. Pour le patient un diagnostic précoce est nécessaire, en raison de la sévérité du syndrome, afin d'adapter le traitement et d'organiser le suivi compte tenu du risque très important de second cancer. La présentation clinique est certes variable mais l'association d'une tumeur du spectre (gliome de haut grade, LNH de type T, cancers colorectaux) à des tâches café au lait ou à des zones hypopigmentées est néanmoins très évocatrice du diagnostic. D’autres éléments cliniques personnels ou familiaux peuvent également évoquer ce diagnostic et un score clinique a été développé par le consortium européen Care for CMMRD (C4CMMRD) (Tableau 1) [4]. Ce score a une très bonne sensibilité pour dépister les patients CMMRD et une consultation de génétique est recommandée pour rechercher ce syndrome chez les patients ayant un score $\geq 3$. Il est donc hautement recommandé d'évoquer un syndrome CMMRD et d'appliquer ce score chez tout enfant ou jeune adulte atteint d'une tumeur digestive, d'une hémopathie de type $T$, ou d'un gliome malin, particulièrement si le patient est originaire de pays où la consanguinité est fréquente.

\section{Analyses moléculaires constitutionnelles}

L'identification de deux variants délétères bi-alléliques d'un des quatre gènes MMR signe le diagnostic de syndrome CMMRD. Cette identification est compliquée du fait à la fois d'une proportion importante de variants de signification inconnue identifiés et de la difficulté technique d'analyse du gène PMS2 qui compte de nombreux pseudogènes [22-24]. Le développement des techniques de séquençage de nouvelle génération permet l'analyse 
conjointe de ces gènes en panel pour un résultat plus rapide mais reste néanmoins soumis à des difficultés techniques pour PMS2 dont l'analyse doit être exhaustive et réalisée dans des laboratoires référents.

L'analyse en panel permet également d'analyser, dans le même temps, d'autres gènes de prédisposition dont certains sont responsables des différents diagnostics différentiels du syndrome CMMRD (TP53, POLE, POLD1, APC...).

D'autres techniques permettent d'étayer le diagnostic de CMMRD et sont utilisées en complément lorsque les analyses constitutionnelles évoquées ci-dessus ne permettent pas de poser avec certitude le diagnostic (identification de variants de signification inconnue, identification d'un seul variant pathogène...).

\section{Immunohistochimie des protéines MMR}

L'observation d'une perte d'expression de l'une (ou deux) des protéines MMR, en immunohistochimie (IHC), non seulement dans la tumeur comme dans le syndrome de Lynch, mais également dans le tissu sain est un élément extrêmement évocateur d'un syndrome CMMRD [4,5]. Cependant, l'absence de marquage dans le tissu sain peut être interprétée à tort comme un échec technique. Les pathologistes doivent être avertis de cela et utiliser un contrôle de marquage à partir d'un autre individu. L'immunohistochimie peut donc aider à différencier les deux syndromes. De plus elle permet de guider les explorations constitutionnelles vers l'un ou l'autre des gènes MMR. Lorsqu'il n'existe pas de matériel disponible pour une étude en IHC, par exemple dans le cas d'hémopathies ou lorsque le diagnostic est suspecté chez une personne indemne de cancer, l'immunomarquage peut être réalisé à partir d'une biopsie de peau [5]. Comme dans le syndrome de Lynch, des mutations tronquantes bi-alléliques de PMS2 ou de MSH6 induisent habituellement une perte d'expression isolée de ces protéines en IHC, alors que les mutations des gènes MLH1 ou MSH2 sont le plus souvent caractérisées par une perte conjointe des protéines MLH1/PMS2 ou MSH2/MSH6 car MLH1 et MSH2 sont des partenaires obligatoires de ces hétérodimères. Il faut souligner que dans le cas de mutations faux sens d'un gène, l'expression de la protéine correspondante peut être conservée, ce qui est une limite de cette analyse dont il convient d'être conscient, puisque dans le cadre des CMMRD, $30 \%$ des variants identifiés sont de type faux sens $[3,19]$. 


\section{Recherche d'instabilité des microsatellites sur les tumeurs ou au niveau}

\section{constitutionnel}

L'étude d'un panel de microsatellites mono et di nucléotidiques, tel que celui utilisé pour le diagnostic du Syndrome de Lynch est tout à fait adaptée à la détection d'un phénotype MSI (Microsatellite instability) dans les tumeurs du spectre du Syndrome de Lynch développées par les patients atteints de syndrome CMMRD. Ce phénotype tumoral ne permet pas de différencier un syndrome de Lynch d'un CMMRD. L'analyse standard des microsatellites n'est en revanche pas adaptée à l'analyse des autres types tumoraux observés dans le syndrome CMMRD, en particulier des tumeurs cérébrales dont plus de $60 \%$ apparaissent stables avec cette technique [3,5]. Or ces tumeurs cérébrales surviennent généralement plus précocement que les tumeurs du spectre du Syndrome de Lynch. Un résultat stable (phénotype MSS), sur une tumeur cérébrale étudiée avec le panel de microsatellites utilisés dans le Syndrome de Lynch ne permet donc pas d'exclure le diagnostic de syndrome CMMRD.

De façon théorique, la présence de mutations constitutionnelles bi-alléliques devrait être associée à une instabilité des microsatellites dans le tissu sain des patients CMMRD. Cette instabilité n'est cependant présente que dans une petite proportion des cellules et ne peut pas être mise en évidence par l'analyse des microsatellites telle qu'elle est faite sur les tumeurs. Un test simple appelé gMSI (germinal microsatellite instability) a été décrit pour détecter cette instabilité dans les tissus non néoplasiques [25]. II repose sur l'analyse des pics surnuméraires généralement associés aux produits de PCR des microsatellites. Sa principale limite est qu'il utilise des microsatellites di-nucléotidiques et, par conséquent, qu'il est inadapté pour détecter une déficience due aux mutations bi-alléliques du gène MSH6 [21,25]. En effet, MSH6 intervient dans la prise en charge des mésappariements impliquant des mono-nucléotides.

\section{Autres méthodes diagnostiques}

Le diagnostic définitif de syndrome CMMRD nécessite l'identification de deux variants délétères bi-alléliques (c'est-à-dire dont la position en trans a été démontrée) constitutionnels d'un des gènes MMR. Malheureusement le taux élevé de VSI et les difficultés d'analyses du gène PMS2 compliquent ce diagnostic [22-24,26]. 
Pour permettre d'arriver à une conclusion dans les cas douteux, deux tests fonctionnels, réalisés à partir des lymphocytes immortalisés des patients, ont été développés [21]. Ces deux tests, basés pour l'un sur l'évaluation de l'instabilité des microsatellites, pour l'autre sur l'étude de la tolérance à la méthylation des lymphocytes immortalisés permettent de confirmer ou d'infirmer le diagnostic de CMMRD dans la plupart des situations. Si les deux tests sont anormaux, le diagnostic est confirmé ; s'ils sont normaux il est exclu, avec une sensibilité et une spécificité proche de $100 \%$. Ces tests sont disponibles en routine en France et peuvent être utilisés dans les situations où les analyses constitutionnelles n'ont pas permis de conclure alors qu'il existe une présentation clinique évocatrice de CMMRD. Ces tests fonctionnels peuvent également être utilisés si aucune tumeur n'est disponible pour les analyses somatiques ou pour tester la fratrie d'un enfant atteint si les deux mutations constitutionnelles n'ont pas pu être identifiées, rendant impossible la réalisation de tests ciblés dans la famille.

Récemment, un taux élevé de mutations somatiques, de l'ordre de 100 mutations par megabase (MB), a été décrit dans les tumeurs des patients atteints d'un syndrome CMMR (versus < 10 mutations /MB pour la plupart des cancers pédiatriques) [8]. Ce phénotype " ultramutateur » semble très caractéristique et, du moins dans les tumeurs cérébrales, il est associé à des mutations somatiques des gènes des polymérases [27]. Son identification devrait jouer un rôle dans les arguments diagnostiques de ce syndrome mais également dans la prise en charge (voir plus loin). Cependant ce phénotype n'est pas spécifique du syndrome CMMRD et a également été observé dans les tumeurs survenant dans le cadre des polyposes associées aux mutations constitutionnelles des gènes POLE ou POLD1 [28]. La différence entre ces deux syndromes de prédisposition peut d'ailleurs aussi être compliquée du fait de l'existence de tumeur MSI dans les tumeurs survenant dans ce contexte de Polymerase Proofreading Associated Polyposis [29].

En pratique, les résultats de l'étude de l'expression des protéines MMR en IHC au niveau de la tumeur et du tissu sain, de l'analyse MSI sur les tissus tumoraux et normaux (gMSI), des tests fonctionnels et de la détermination de la charge mutationnelle tumorale constituent un faisceau d'arguments diagnostiques permettant de suspecter voire de confirmer le diagnostic de syndrome CMMRD, en particulier lorsqu'il n'est pas clairement établi par les 
analyses constitutionnelles. Toutes ces techniques ont cependant leurs limites et leur combinaison est souvent nécessaire pour affirmer ou exclure le diagnostic.

\section{Pris? ?n charg?}

\section{Conseil génétique}

Une consultation d'oncogénétique doit être proposée aux patients et à leurs parents lorsque le diagnostic de syndrome CMMRD est suspecté sur des éléments cliques et/ou somatiques (résultats de I'analyse MSI et/ou de I'IHC, voire de la charge mutationnelle). Lors de cette consultation, une information complète est délivrée et un consentement signé. Sont explicités les objectifs et les limites de l'analyse moléculaire proposée chez l'enfant atteint, ainsi que l'obligation d'information des apparentés en cas de variant(s) délétère(s) identifié(s). On discutera également les diagnostics différentiels possibles. Compte tenu de la sévérité de ce syndrome, un soutien psychologique doit systématiquement être proposé au patient et à sa famille. Ils doivent être informés des implications thérapeutiques potentielles du résultat du test ainsi que du risque élevé d'une deuxième malignité en cas de confirmation du diagnostic de syndrome CMMRD.

Lors de cette première consultation ainsi que lors de la consultation de restitution du résultat si le diagnostic est confirmé, doivent également être discutés le risque potentiel de récurrence de $25 \%$ chez les frères et sœurs, et les risques de cancer associés au Syndrome de Lynch chez les porteurs hétérozygotes, en particulier chez les deux parents, porteurs obligatoires. Des mesures de surveillance telles que recommandées dans le Syndrome de Lynch leur sont alors proposées.

L'identification des deux altérations bi-alléliques constitutionnelles est importante pour offrir aux familles des patients CMMRD des conseils appropriés, discuter avec eux de la réalisation de tests prédictifs ciblés mais aussi des possibilités de diagnostic prénatal/préimplantatoires.

\section{Surveillance des patients CMMRD}

Les deux principaux groupes internationaux s'intéressant à cette pathologie (le consortium européen Care for CMMRD (C4CMMRD) et le consortium international: International BMMRD Consortium) ont proposé des protocoles de surveillance pour les patients CMMRD 
basés sur les données disponibles concernant le type et la fréquence des tumeurs ainsi que leurs âges de survenue [5,30,31]. La surveillance digestive, hématologique et neurologique commence dès l'enfance et s'y ajoute une surveillance gynécologique et urinaire à partir de 20 ans (Table 2). Les recommandations des deux consortiums sont très similaires et soulignent les mêmes points. L'efficacité de la surveillance cérébrale en termes de diagnostic précoce ou de curabilité des lésions n'est pas connue et reste à évaluer. De même, bien que les hémopathies soient les troisièmes atteintes malignes en fréquence, et les plus précoces chez les enfants atteints de CMMRD, le manque actuel d'outils efficaces de dépistage limite les recommandations de dépistage. Néanmoins, la réalisation d'une échographie abdominale et d'une numération tous les 6 mois a été évoquée [30,32]. La numération a l'intérêt également de rechercher une anémie. La surveillance digestive en revanche, permet l'identification et la résection de polypes adénomateux et ainsi de prévenir, dans une certaine mesure, le cancer colorectal. Le consortium international BMMRD a publié en 2012 des recommandations de suivi digestif par coloscopie annuelle à partir de l'âge de 3 ans (5 ans avant le plus jeune cas de CCR décrit chez un patient CMMRD) puis en 2016 la même équipe recommande finalement de commencer cette surveillance à 6 ans $[10,31]$. Le consortium européen recommande pour sa part des coloscopies annuelles à partir de 8 ans [30]. Le rythme annuel doit être rapproché à un examen tous les 6 mois à partir du moment où des polypes ont été identifiés. Comme dans le Syndrome de Lynch et dans les diverses polyposes colorectales atténuées, l'utilisation d'une coloration à l'indigo-carmin peut probablement aider à mieux estimer le nombre de polypes en particulier en améliorant la détection des polypes plans, mais son bénéfice n'a pas été évalué dans le contexte rare du syndrome CMMRD. Les patients ayant des adénomes en dysplasie de haut garde sont à très haut risque de cancer colique. Une prise en charge chirurgicale, à type de colectomie subtotale avec anastomose iléo-rectale ou une coloproctectomie, doit être discutée en fonction du phénotype colique (nombre de polypes, taille, degré de dysplasie). Une évaluation soigneuse du rectum précisant son atteinte est nécessaire pour la décision de sa conservation ou non. Ce type de décision peut être compliqué par la prévalence élevée des polypes ou des cancers du tractus digestif supérieur ou du grêle. L'âge d'apparition de ce type de lésions semble un peu plus tardif que celui des adénomes coliques. L'exploration endoscopique haute et une surveillance par vidéocapsule sont recommandées annuellement à partir de l'âge de 8 ou 10 ans [30]. 
Une suggestion du consortium IBBMRD, reprise par un autre groupe d'expert ensuite, est d'ajouter à cette surveillance une IRM corps entier annuelle à partir de 6 ans (ou dès que l'on peut se passer d'une anesthésie générale), comme recommandé au cours du syndrome de Li Fraumeni [5, 31, 32]. Cependant cet examen ne pourrait pas remplacer l'IRM cérébrale qui est plus sensible pour la détection des lésions du système nerveux central.

L'efficacité et l'acceptabilité de ces modalités de surveillance sont encore inconnues et restent à évaluer. La lourdeur de cette surveillance conduit cependant à pressentir que son acceptabilité sera probablement difficile.

\section{Traitement}

Le rôle de la chimiothérapie et de la radiothérapie dans la survenue de secondes tumeurs chez les patients CMMRD a été évoqué mais est difficile à établir et il y existe très peu de données disponibles concernant la toxicité de ces traitements chez ces patients.

Plusieurs agents chimiothérapeutiques courants nécessitent une réparation adéquate des mésappariements pour exercer leur activité anti-tumorale. Il s'agit notamment du cas de la mercaptopurine et du témozolomide qui sont couramment utilisés dans le traitement des hémopathies et des gliomes. II a été démontré que les cellules déficientes pour le système MMR étaient plus tolérantes au témozolomide et à la radiothérapie que les cellules MMRcompétentes [33-35]. Il est important de noter qu'il n'y a pas de baisse d'efficacité rapportée avec d'autres agents thérapeutiques tels que d'autres alkylants et les anthracyclines. Cela doit nous inciter à concevoir des protocoles spécifiques pour les tumeurs associées au CMMRD, ce qui implique encore une fois, d'en faire le diagnostic précocement.

Enfin, le phénotype " ultramutateur » décrit dans certaines tumeurs liées au CMMRD offre l'opportunité de nouvelles approches pour le traitement de ces patients [36]. En effet, les inhibiteurs des checkpoints immunitaires, connus pour être efficaces sur les tumeurs dans le cadre du syndrome de Lynch, ont montré un effet significatif en termes de réponse tumorale et de survie chez deux patients atteints de tumeurs gliales de haut grade en récidive [37]. Cette observation souligne l'importance d'un diagnostic précoce qui permettra d'inclure ces patients dans des essais afin d'évaluer l'efficacité de ces traitements d'immunothérapie. Des essais spécifiques pour les tumeurs associées à ce syndrome sont déjà ouverts aux inclusions. Le séquençage systématique de ces tumeurs permettrait également d'identifier 
des mutations dans des gènes cibles pour potentiellement offrir une approche thérapeutique ciblée à ces patients.

La possibilité de chimioprévention est probablement l'élément le plus important dans ce contexte de prédisposition à très forte pénétrance. Plusieurs options ont été suggérées telle que l'utilisation des anti-inflammatoires comme l'aspirine, qui est connue pour réduire le risque de cancer colique dans le syndrome de Lynch $[38,39]$ ou l'acide rétinoïque, et plus récemment les inhibiteurs des checkpoints immunitaires $[36,40]$. La possibilité de vaccination, déjà à l'étude dans le Syndrome de Lynch, est également à garder à l'esprit [41]. Néanmoins ces différentes options prometteuses restent à évaluer dans le cadre d'essais prospectifs.

\section{Conclusion}

En raison de sa présentation clinique variable et du chevauchement phénotypique avec d'autres syndromes de prédisposition (NF1, syndrome de Li-Fraumeni, polyposes adénomateuses colorectales), le syndrome CMMRD est souvent méconnu par les cliniciens. Une meilleure connaissance des critères cliniques et des méthodes diagnostiques propres à ce syndrome devrait permettre d'augmenter le nombre de patients identifiés au moment de leur première tumeur et même avant [42]. Cela permettra d'ajuster les modalités de traitement et de mettre en place des stratégies de surveillance spécifiques pour détecter d'autres tumeurs malignes non seulement chez le patient, mais aussi chez ses frères et sœurs porteurs de mutations bi-alléliques. Le pronostic de ce syndrome est encore très sombre et dans le contexte de sa rareté, seule une collecte et une évaluation systématiques de toutes les données cliniques disponibles permettront d'améliorer la prise en charge de ces patients. Par conséquent, nous encourageons les cliniciens à signaler les patients CMMRD qu'ils prennent en charge aux registres tel que celui établi par le consortium européen C4CMMRD.

\section{Référ??nc? ??}

1. Wang Q, Lasset C, Desseigne F, Frappaz D, Bergeron C, et al. Neurofibromatosis and early onset of cancers in hMLH1-deficient children. Cancer Res. 1999 Jan 15;59(2):294-7. 
2. Ricciardone MD, Ozçelik $T$, Cevher $B$, Ozdağ $H$, Tuncer $M$, et al. Human MLH1 deficiency predisposes to hematological malignancy and neurofibromatosis type 1 . Cancer Res. 1999 Jan 15;59(2):290-3.

3. Lavoine N, Colas C, Muleris M, Bodo S, Duval A, et al. Constitutional mismatch repair deficiency syndrome: clinical description in a French cohort. J Med Genet. 2015 Nov;52(11):770-8.

4. Wimmer K, Kratz CP, Vasen HFA, Caron O, Colas C, et al. Diagnostic criteria for constitutional mismatch repair deficiency syndrome: suggestions of the European consortium 'care for CMMRD' (C4CMMRD). J Med Genet. 2014 Jun;51(6):355-65.

5. Bakry D, Aronson M, Durno C, Rimawi H, Farah R, et al. Genetic and clinical determinants of constitutional mismatch repair deficiency syndrome: report from the constitutional mismatch repair deficiency consortium. Eur J Cancer Oxf Engl 1990. 2014 Mar;50(5):987-96.

6. Turcot J, Despres JP, St Pierre F. Malignant tumors of the central nervous system associated with familial polyposis of the colon: report of two cases. Dis Colon Rectum. 1959 Oct;2:465-8.

7. Hamilton SR, Liu B, Parsons RE, Papadopoulos N, Jen J, et al. The molecular basis of Turcot's syndrome. N Engl J Med. 1995 Mar 30;332(13):839-47.

8. Shlien A, Campbell BB, de Borja R, Alexandrov LB, Merico D, et al. Combined hereditary and somatic mutations of replication error repair genes result in rapid onset of ultra-hypermutated cancers. Nat Genet. 2015 Mar;47(3):257-62.

9. Ripperger T, Schlegelberger B. Acute lymphoblastic leukemia and lymphoma in the context of constitutional mismatch repair deficiency syndrome. Eur J Med Genet. 2016 Mar;59(3):133-42.

10. Aronson M, Gallinger S, Cohen Z, Cohen S, Dvir R, et al. Gastrointestinal Findings in the Largest Series of Patients With Hereditary Biallelic Mismatch Repair Deficiency Syndrome: Report from the International Consortium. Am J Gastroenterol. 2016 Feb;111(2):275-84.

11. Wimmer K, Beilken A, Nustede R, Ripperger T, Lamottke B, et al. A novel germline POLE mutation causes an early onset cancer prone syndrome mimicking constitutional mismatch repair deficiency. Fam Cancer. 2017 Jan;16(1):67-71.

12. Chmara $M$, Wernstedt A, Wasag B, Peeters $H$, Renard $M$, et al. Multiple pilomatricomas with somatic CTNNB1 mutations in children with constitutive mismatch repair deficiency. Genes Chromosomes Cancer. 2013 Jul;52(7):656-64.

13. Wimmer K, Rosenbaum T, Messiaen L. Connections between constitutional mismatch repair deficiency syndrome and neurofibromatosis type 1. Clin Genet. 2017 Apr;91(4):50719. 
14. Gardès $P$, Forveille $M$, Alyanakian $M-A$, Aucouturier $P$, llencikova $D$, et al. Human MSH6 deficiency is associated with impaired antibody maturation. J Immunol Baltim Md 1950. 2012 Feb 15;188(4):2023-9.

15. Crouse GF. Non-canonical actions of mismatch repair. DNA Repair. 2016 Feb;38:1029.

16. Tesch VK, IJspeert H, Raicht A, Rueda D, Dominguez-Pinilla N, et al. No Overt Clinical Immunodeficiency Despite Immune Biological Abnormalities in Patients With Constitutional Mismatch Repair Deficiency. Front Immunol. 2018 Jul 2;9:1506.

17 Win AK, Jenkins MA, Dowty JG, Antoniou AC, Lee A, et al. Prevalence and Penetrance of Major Genes and Polygenes for Colorectal Cancer. Cancer Epidemiol Biomark Prev Publ Am Assoc Cancer Res Cosponsored Am Soc Prev Oncol. 2017 Mar;26(3):404-12.

18 Oncogénétique en 2016 /consultations, laboratoires et suivi, INCa, décembre 2017

19 Felton KEA, Gilchrist DM, Andrew SE. Constitutive deficiency in DNA mismatch repair. Clin Genet. 2007 Jun;71(6):483-98.

20 Li L, Hamel N, Baker K, McGuffin MJ, Couillard M, et al. A homozygous PMS2 founder mutation with an attenuated constitutional mismatch repair deficiency phenotype. J Med Genet. 2015 May;52(5):348-52.

21 Bodo S, Colas C, Buhard O, Collura A, Tinat J, et al. Diagnosis of Constitutional Mismatch Repair-Deficiency Syndrome Based on Microsatellite Instability and Lymphocyte Tolerance to Methylating Agents. Gastroenterology. 2015 Oct;149(4):1017-1029.e3.

22 Wimmer K, Wernstedt A. PMS2 gene mutational analysis: direct cDNA sequencing to circumvent pseudogene interference. Methods Mol Biol Clifton NJ. 2014;1167:289-302.

23 Vaughn CP, Hart KJ, Samowitz WS, Swensen JJ. Avoidance of pseudogene interference in the detection of 3' deletions in PMS2. Hum Mutat. 2011 Sep;32(9):1063-71.

24 van der Klift HM, Mensenkamp AR, Drost M, Bik EC, Vos YJ, et al. Comprehensive Mutation Analysis of PMS2 in a Large Cohort of Probands Suspected of Lynch Syndrome or Constitutional Mismatch Repair Deficiency Syndrome. Hum Mutat. 2016 Nov;37(11):116279.

25 Ingham D, Diggle CP, Berry I, Bristow CA, Hayward BE, et al. Simple Detection of Germline Microsatellite Instability for Diagnosis of Constitutional Mismatch Repair Cancer Syndrome. Hum Mutat. 2013 Jun 1;34(6):847-52.

26 Vogt J, Wernstedt A, Ripperger T, Pabst B, Zschocke J, et al. PMS2 inactivation by a complex rearrangement involving an HERV retroelement and the inverted 100-kb duplicon on 7p22.1. Eur J Hum Genet EJHG. 2016 Nov;24(11):1598-604.

27. Andrianova MA, Chetan GK, Sibin MK, Mckee T, Merkler D, et al. Germline PMS2 and somatic POLE exonuclease mutations cause hypermutability of the leading DNA strand in 
biallelic mismatch repair deficiency syndrome brain tumours. J Pathol. 2017 Nov;243(3):331341.

28. Campbell BB, Light N, Fabrizio D, Zatzman M, Fuligni F, et al. Comprehensive Analysis of Hypermutation in Human Cancer. Cell. 2017 Nov 16;171(5):1042-1056

29. Elsayed FA, Kets CM, Ruano D, van den Akker B, Mensenkamp AR, et al.Germline variants in POLE are associated with early onset mismatch repair deficient colorectal cancer. Eur J Hum Genet. 2015 Aug;23(8):1080-4

30 Vasen HFA, Ghorbanoghli Z, Bourdeaut F, Cabaret O, Caron O, et al. Guidelines for surveillance of individuals with constitutional mismatch repair-deficiency proposed by the European Consortium 'Care for CMMR-D' (C4CMMR-D). J Med Genet. 2014 May;51(5):28393.

31 Durno C, Boland CR, Cohen S, Dominitz JA, Giardiello FM, et al. Recommendations on Surveillance and Management of Biallelic Mismatch Repair Deficiency (BMMRD) Syndrome: A Consensus Statement by the US Multi-Society Task Force on Colorectal Cancer. Gastroenterology. 2017 May;152(6):1605-1614

32. Tabori U, Hansford JR, Achatz MI, Kratz CP, Plon SE, et al. Clinical Management and Tumor Surveillance Recommendations of Inherited Mismatch Repair Deficiency in Childhood. Clin Cancer Res. 2017 Jun 1;23(11):e32-e37

33 Karran P, Attard N. Thiopurines in current medical practice: molecular mechanisms and contributions to therapy-related cancer. Nat Rev Cancer. 2008 Jan;8(1):24-36.

34 Ilencikova D, Sejnova D, Jindrova J, Babal P. High-grade brain tumors in siblings with biallelic MSH6 mutations. Pediatr Blood Cancer. 2011 Dec 1;57(6):1067-70.

35 Hunter C, Smith R, Cahill DP, Stephens P, Stevens C, et al. A hypermutation phenotype and somatic MSH6 mutations in recurrent human malignant gliomas after alkylator chemotherapy. Cancer Res. 2006 Apr 15;66(8):3987-91.

36. Westdorp $\mathrm{H}$, Kolders $\mathrm{S}$, Hoogerbrugge $\mathrm{N}$, de Vries IJM, Jongmans $\mathrm{MCJ}$,. Immunotherapy holds the key to cancer treatment and prevention in constitutional mismatch repair deficiency (CMMRD) syndrome. Cancer Lett. 2017 Sep 10;403:159-164.

37 Bouffet E, Larouche V, Campbell BB, Merico D, de Borja R, et al. Immune Checkpoint Inhibition for Hypermutant Glioblastoma Multiforme Resulting From Germline Biallelic Mismatch Repair Deficiency. J Clin Oncol Off J Am Soc Clin Oncol. 2016 Mar 21;

38 Burn J, Gerdes A-M, Macrae F, Mecklin J-P, Moeslein G, et al. Long-term effect of aspirin on cancer risk in carriers of hereditary colorectal cancer: an analysis from the CAPP2 randomised controlled trial. Lancet Lond Engl. 2011 Dec 17;378(9809):2081-7.

39 Leenders EKSM, Westdorp H, Brüggemann RJ, Loeffen J, Kratz C, et al. Cancer prevention by aspirin in children with Constitutional Mismatch Repair Deficiency (CMMRD). Eur J Hum Genet. 2018 June 
40 Gottschling S, Reinhard H, Pagenstecher C, Krüger S, Raedle J, et al. Hypothesis: Possible role of retinoic acid therapy in patients with biallelic mismatch repair gene defects. Eur J Pediatr. 2008 Feb;167(2):225-9.

41. von Knebel Doeberitz $M$, Kloor $M$. Towards a vaccine to prevent cancer in Lynch syndrome patients. Fam Cancer. 2013 Jun;12(2):307-12

42. Suerink $M$, Potjer TP, Versluijs $A B$, Ten Broeke SW, et al. Constitutional mismatch repair deficiency in a healthy child: On the spot diagnosis? Clin Genet. 2018 Jan;93(1):134137 


\section{Lég??nd? $\mathbf{s}$ d? ?ls figur? ?s}

Figure 1:

Exemples de différentes présentations familiales de patients CMMRD (données personnelles C Colas)

Noir: patients atteints de tumeurs dans le cadre d'un syndrome CMMRD; hachurés: patients atteints de tumeurs dans le cadre d'un syndrome de Lynch ; (âge au diagnostic) ; Ad : adénome colique ; LAM: leucémie aigüe myéloblastique ; CCR : cancer colorectal ; TCLs: taches café au lait; TC: tumeur cérébrale; CD: cancer duodénal; CE : cancer de I'endomètre ; VD : variant délétère, VSI variant de signification inconnue

Figure 2

Répartitions des types tumoraux et de leurs âges au diagnostic dans le syndrome CMMRD (données françaises) [3]

LAM : leucémie aigüe myéloblastique, Ly B : lymphome lymphoblastique de type B, Ly T: lymphome lymphoblastique de type $\mathrm{T}$, SNC : système nerveux central

Figure 3

Différence de répartitions des gènes impliqués dans le syndrome CMMRD et dans le syndrome de Lynch

\section{Conflit d'intérêt : aucun}



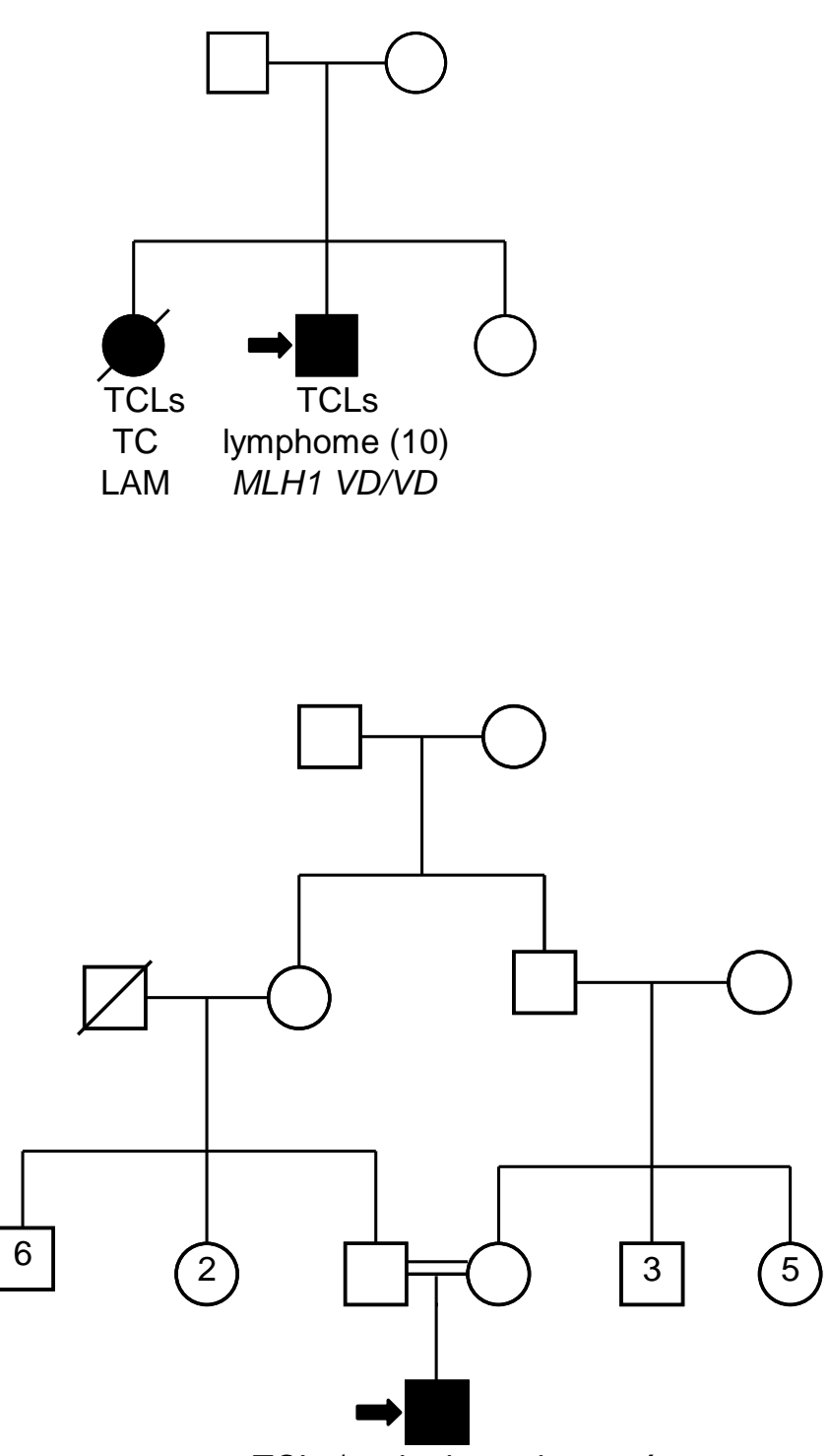

TCLs/ tache hypopigmentée Lymphome T 8

$$
\text { TC14 }
$$

2 CCR + multiples ad 14 MSH6 VUS/VUS

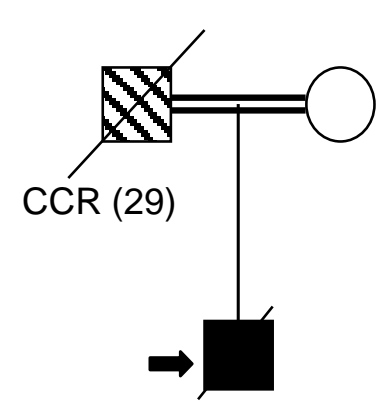

TCLs / cavernome lymphome (3) MSH2 VD/VD

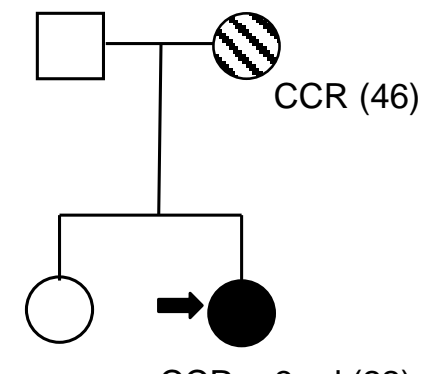

$\mathrm{CCR}+3$ ad (22)

$3 \mathrm{ad}(23) / 1$ ad (24)

TC (34)

CE (36)

CD (36)

PMS2 VD/VD

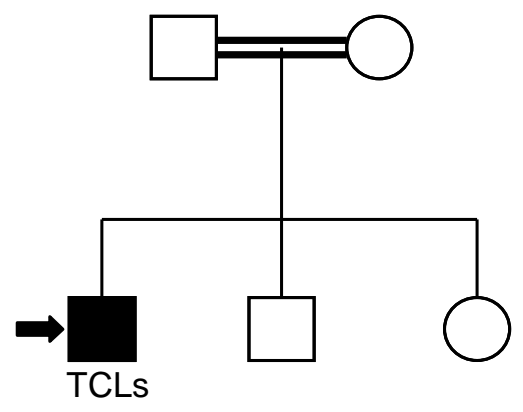

Lymphome 5

CCR 16

PMS2 VD/VD

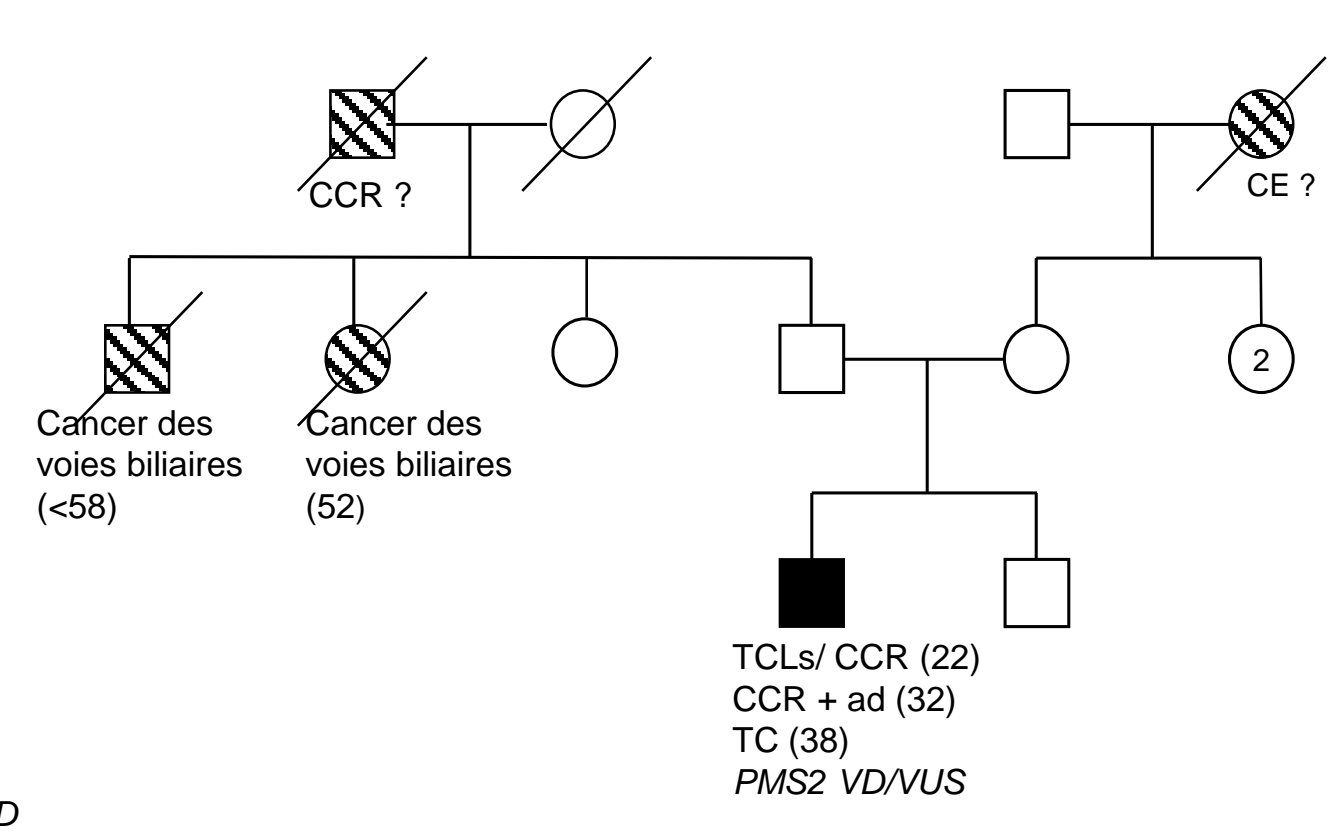

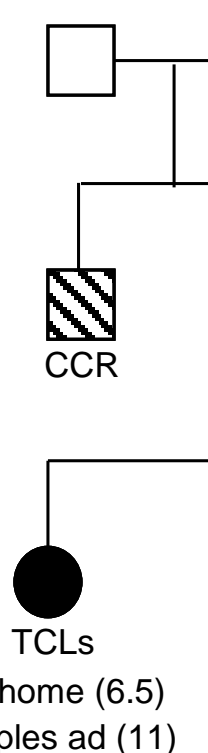

multiples ad (11)

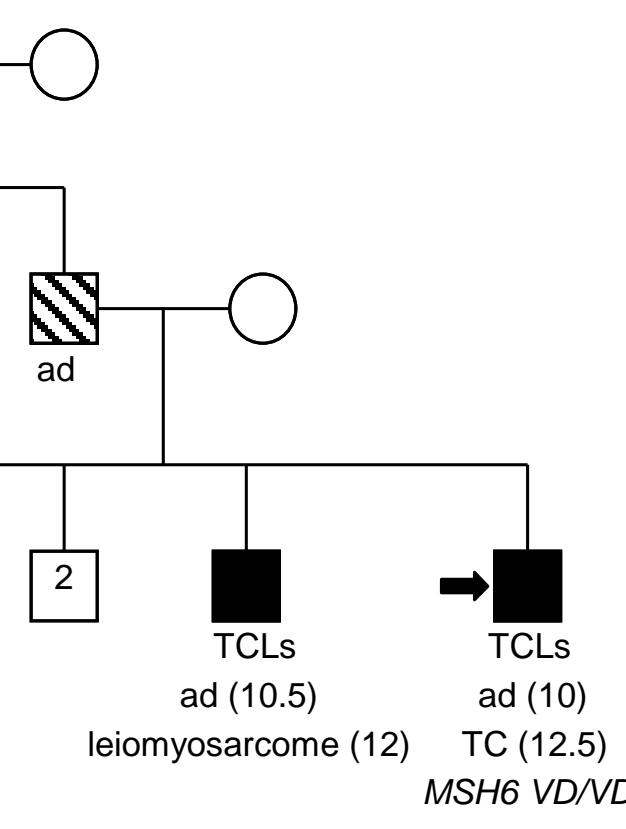




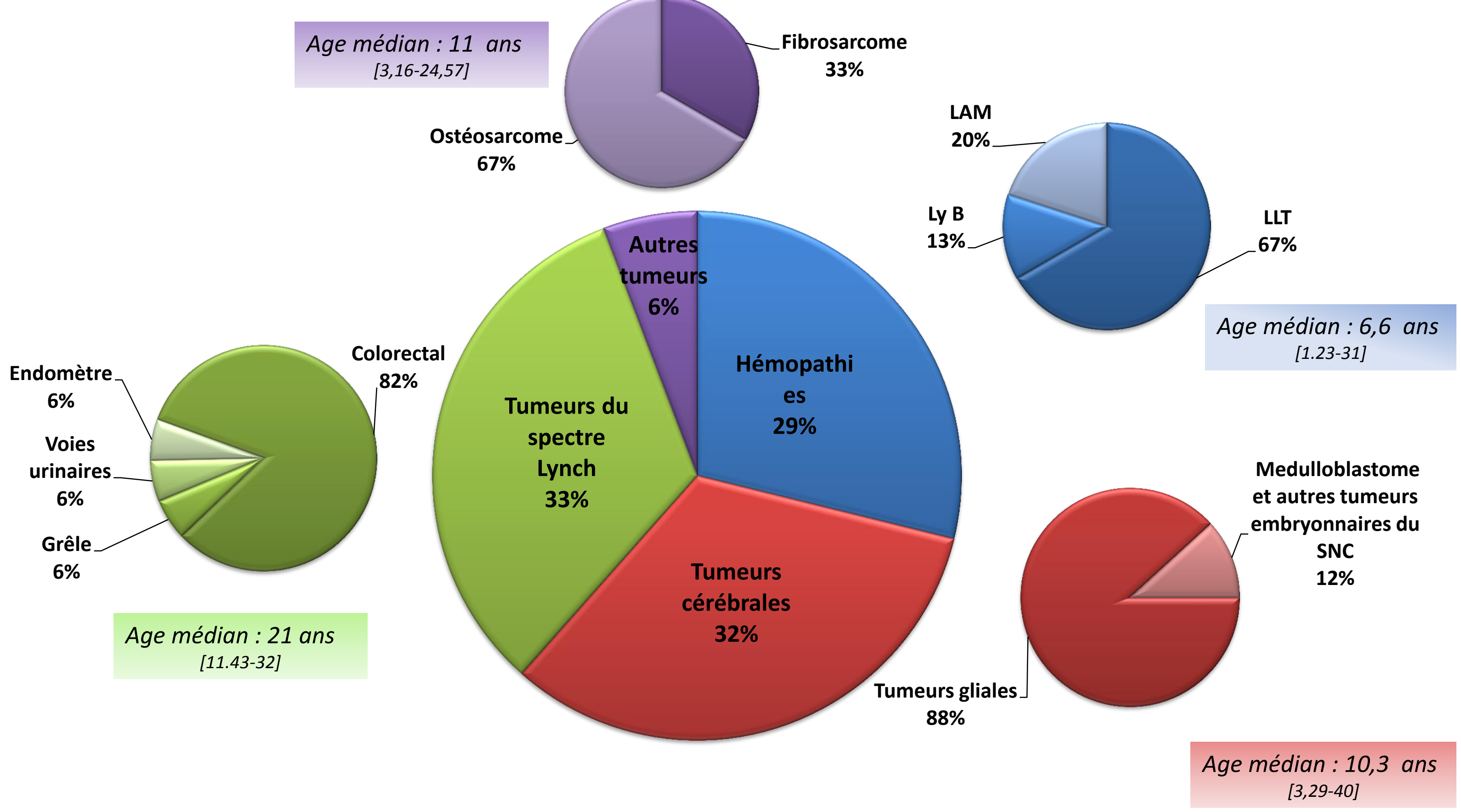




\section{Syndrome CMMRD}

Source : base de données européenne C4CMMRD

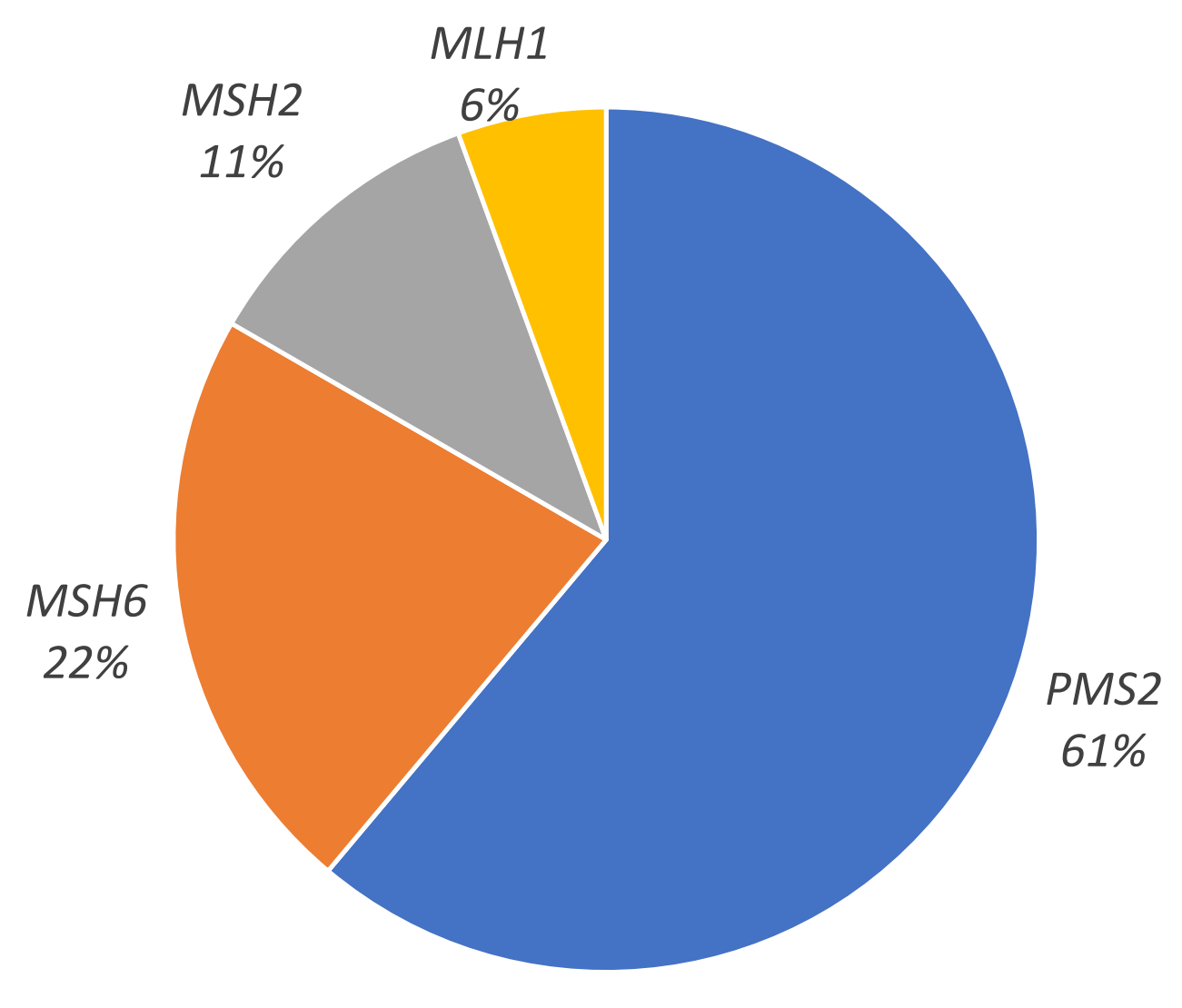

\section{Syndrome de Lynch}

Source : Oncogénétique en 2016 /consultations, laboratoires et suivi, INCa, décembre 2017 [18]

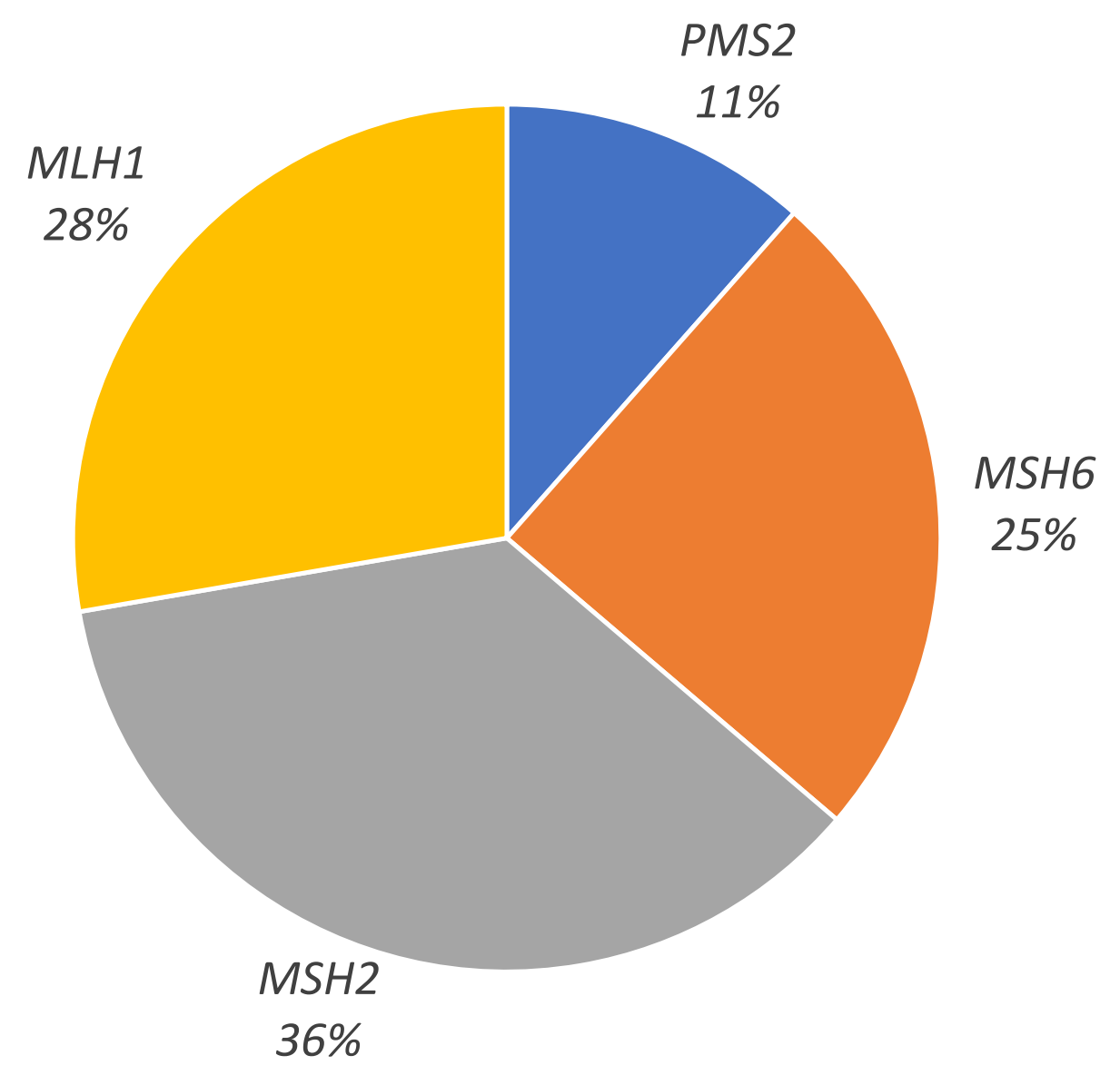


Tableau 1

Score clinique permettant d'évoquer le diagnostic de syndrome CMMD.

Adapté de Wimmer et al. J Med Genet 2014 [4]

\begin{tabular}{|c|c|}
\hline Indications de recherche de syndrome CMMRD chez les patients atteints de cancer & $\mathrm{Si} \geq 3$ points \\
\hline \multicolumn{2}{|l|}{ Cancers ou lésions précancéreuses : } \\
\hline \multicolumn{2}{|l|}{ au moins un item requis ; si plus d'un additionner les points } \\
\hline Cancer du spectre du SL* $<25$ ans & 3 points \\
\hline $\begin{array}{l}\text { Adénomes coliques multiples }<25 \text { ans (sans mutation APC/MUTYH) ou un adénome } \\
\text { unique en dysplasie sévère }<25 \text { ans }\end{array}$ & 3 points \\
\hline Gliome de haut grade (III ou IV) $<25$ ans & 2 points \\
\hline LNH de type T ou tumeur embryonnaire du SNC $<18$ ans & 2 points \\
\hline Tout autre cancer $<18$ ans & 1 point \\
\hline \multicolumn{2}{|l|}{ Autres éléments: } \\
\hline \multicolumn{2}{|l|}{ Optionnels; si plus d'un item additionner les points } \\
\hline Signes cliniques de NF1 et/ou $\geq 2$ taches café-au lait et/ou dépigmentées $\varnothing>1 \mathrm{~cm}$ & 2 points \\
\hline Diagnostic d'un SL chez un apparenté du $1^{\text {er }}$ ou $2^{\text {nd }}$ degré & 2 points \\
\hline Cancer du spectre du $S L^{*}<60$ ans chez un apparenté du $1^{\text {er }}, 2^{\text {nd }}$ ou $3^{\text {ème }}$ degré & 1 point \\
\hline $\begin{array}{l}\text { Un frère ou une sœur avec un cancer du spectre du } \mathrm{SL}^{*} \text {, un gliome de haut grade, } \\
\text { une tumeur embryonnaire du SNC ou un LNH }\end{array}$ & 2 points \\
\hline Un frère ou une sœur avec tout autre cancer $<18$ ans & 1 point \\
\hline Pilomatricomes multiples chez le patient & 2 points \\
\hline Pilomatricome unique chez le patient & 1 point \\
\hline Agénésie du corps calleux ou cavernome primitif chez le patient & 1 point \\
\hline Parents consanguins & 1 point \\
\hline Déficit ou taux réduits d'IgG2/4 et/ou d IgA & 1 point \\
\hline
\end{tabular}

*Côlon/rectum, endomètre, intestin grêle, voies urinaires, voies biliaires, estomac, ovaire.

CMMRD : constitutional mismatch repair deficiency; SL : Syndrome de Lynch; LNH : Lymphome non-

Hodgkinien ; SNC : système nerveux central 
Tableau 2

Recommandations de suivi pour les patients atteints de syndrome CMMRD

\begin{tabular}{|c|c|c|}
\hline \multirow[t]{2}{*}{ Type d'examen } & \multicolumn{2}{|c|}{ Rythme et âge de début } \\
\hline & $\begin{array}{l}\text { C4CMMRD consortium } \\
\text { européen [30] }\end{array}$ & $\begin{array}{l}\text { BMMRD consortium } \\
\text { international [31] }\end{array}$ \\
\hline Examen clinique & / 6 mois à partir de 1 an & \\
\hline NFS & / 6 mois à partir de 1 an & /6 mois à partir de 1 an \\
\hline Echographie abdominale & $\begin{array}{l}\text { Optionnelle } \\
\text { / } 6 \text { mois à partir de } 1 \text { an }\end{array}$ & \\
\hline IRM cérébrale & $/ 6-12$ mois à partir de 2 ans & $/ 6$ mois à partir de 2 ans \\
\hline Coloscopie & $\begin{array}{l}\text { /an à partir de } 8 \text { ans } \\
/ 6 \text { mois dès que des } \\
\text { adénomes sont identifiés }\end{array}$ & $\begin{array}{l}\text { /an à partir de } 6 \text { ans } \\
/ 6 \text { mois dès que des } \\
\text { adénomes sont identifiés }\end{array}$ \\
\hline Endoscopie haute & /an à partir de 10 ans & /an à partir de 8 ans \\
\hline Examen du grêle par vidéocapsule & /an à partir de 10 ans & /an à partir de 8 ans \\
\hline $\begin{array}{l}\text { Examen gynécologique avec } \\
\text { biopsie d'endomètre }\end{array}$ & /an à partir de 20 ans & /an à partir de 20 ans \\
\hline Echographie pelvienne & /an à partir de 20 ans & /an à partir de 20 ans \\
\hline $\begin{array}{lll}\begin{array}{l}\text { Analyse d'urine } \\
\text { bandelette) }\end{array} & \text { (cytologie }+ \\
\end{array}$ & /an à partir de 20 ans & /an à partir de 10 ans \\
\hline IRM corps entier & & A évaluer \\
\hline
\end{tabular}

